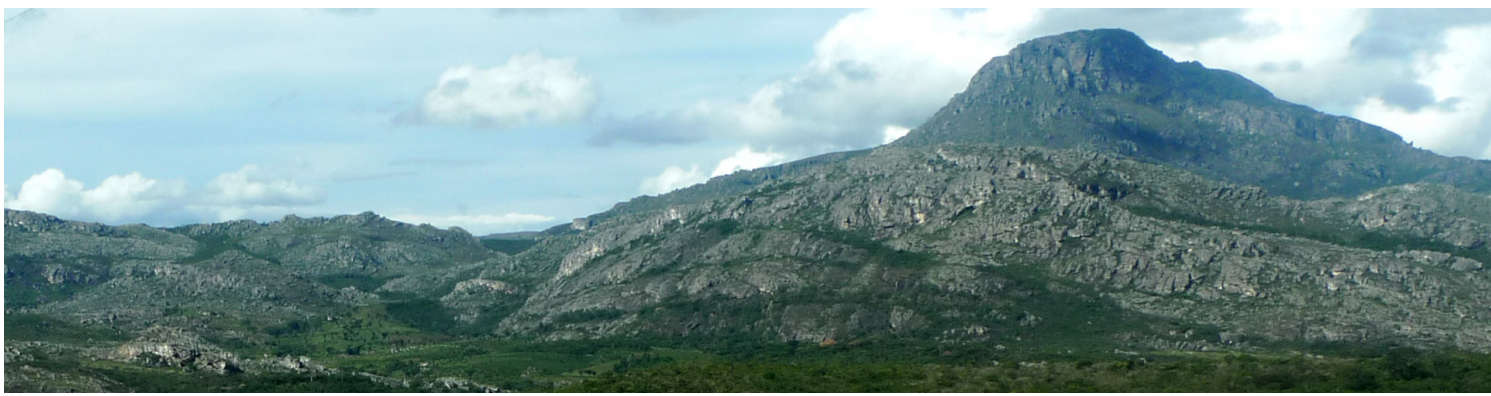

Fonte da imagem: http://csr.ufmg.br/geoespinhaco/img/bota.jpg

\title{
Roteiros de campo em Terræ Didatica para formar geólogos e outros profissionais de Ciências da Terra
}

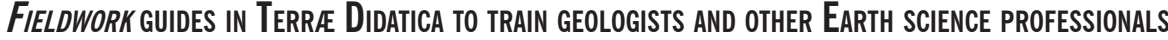 \\ Celso dal Ré Carnerro $\frac{1}{2}$
}

1- Universidade Estadual de Campinas, Programa de Pós-Graduação em Ensino e História de Clências da Terra. Caixa Postal 6152, 13083-970 Campinas, SP, Brasil. 2- BOLSISTA DO CNPQ.E-MALl: CEDREC@IGE.UNICAMP.BR

Abstract: The December 2017 edition of Terræ Didatica includes two field trip guides that interest the subject of academic and professional training of geologists, geological engineers and other professionals in the broad area of Earth Sciences. One of them is briefly analyzed in this commentary, focusing the Serra do Espinhaço Meridional, in the Minas Gerais State (MG). The guide describes visiting points and rock expositions, which are specially important for understanding the stratigraphy and depositional systems of the Espinhaço Supergroup and Bambuí and Macaúbas Groups. Another guide of the same issue shows the relevance of an authentic didactic laboratory that includes open-air exhibitions and underground galleries for student training of courses in Mining or Geology: the Santa Luzia School Mine in the Paraíba State (PB).
Manuscrito:

Recebido: 25/02/2014

Corrigido: $16 / 03 / 2016$

Aceito: 21/03/2016

Citation:Carneiro C.D.R. 2018. Roteiros de campo em Terræ Didatica para formar geólogos e outros profissionais de Ciências da Terra. Terræ Didatica, 14(1):80-84. (Resenha). URL: http://www.ige.unicamp.br/terraedidatica/

\section{Relevância do tema}

Trabalhos de campo são absolutamente essenciais na formação de geólogos, em qualquer parte do mundo. No Brasil, desde os pioneiros cursos de graduação em Geologia dos anos 1950, cada curso manteve alto nível de interesse e atenção com as atividades de campo.

A complexa evolução geológica da magnífica Serra do Espinhaço Meridional, que atravessa a porção centro-norte do Estado de Minas Gerais, desafiou os autores Uhlein et al. (2017) a sistematizar conhecimentos e a compartilhar experiências práticas em um Roteiro de campo sobre a Estratigrafia e os sistemas deposicionais do Supergrupo Espinhaço e Grupos Bambuí e Macaúbas, recentemente publicado por Terræ Didatica. Importante característica do roteiro é a utilização de recursos da mais alta tec- nologia de representação espacial, acompanhados pela descrição pormenorizada de pontos de parada meticulosamente selecionados pelos autores. $\mathrm{O}$ leitor encontra, ao longo do roteiro, indicações cuidadosas de caminhamentos, percursos, mapas, perfis geológicos e esquemas, além de belas fotografias e imagens.

No mesmo número da revista, o trabalho de Santos et al. (2017) focaliza outra preciosidade para atividades de campo de treinamento em cursos técnicos e de graduação, nos campos de Geologia e Mineração: A Mina-Escola de Santa Luzia-PB: um laboratório didático para estudantes de Mineração/ Geologia. Ricamente ilustrado, o trabalho apresenta pormenores das escavações abandonadas pelas atividades de lavra de minérios nas proximidades da cidade de Santa Luzia, Estado da Paraíba e descre- 
ve maneiras de utilizá-las em atividades didáticas.

Os dois artigos ultrapassam, contudo, o mero aspecto da visitação pública proporcionada pelos lugares escolhidos e ganham maior interesse se examinarmos brevemente a história da implantação dos cursos de Geologia no País. Como o foco inicial de Uhlein et al. (2017) está em Diamantina, MG, devemos citar também a criação de um patrimônio intangível que atende há décadas aos cursos de Geologia brasileiros: o Centro de Geologia Eschwege (CGE), implantado em outubro de 1969.

A formação de geólogos foi considerada uma questão de Estado nas décadas de 1940-1950, como consequência da criação de grandes empresas estatais e do processo de industrialização do País (Azevedo \& Terra 2008). Na época, as autoridades entenderam que a introdução de uma carreira de geólogos profissionais interromperia o incansável fluxo de especialistas e consultores estrangeiros e, assim, implantaram a CAGE - Campanha Nacional de Formação de Geólogos (Barroso 1996), em histórica decisão assinada em 18 janeiro de 1957. Há dois meses comemoraram-se 51 anos da divulgação do importante documento, cujos efeitos e desdobramentos os geólogos formados há mais tempo conhecem bem. Cerca de 25 anos depois, nos anos 1980, a comunidade nacional de geólogos e engenheiros geólogos se organizou, sob a égide da Sociedade Brasileira de Geologia e com apoio do Ministério da Educação, para produzir levantamentos e análises sobre o direcionamento, as qualidades e as deficiências da formação profissional nesse campo. Os trabalhos convergiram para a definição do chamado Currículo Mínimo dos cursos (SBG 1983, 1984).

Assine (1994) acentua que a demanda dos profissionais pelo mercado de trabalho é fortemente influenciada pelas mudanças da economia, cujo vigor oscila de acordo com a alternância de períodos de crescimento e recessão econômica. Não obstante, cabe aos geólogos a obrigação de gerar os insumos minerais e energéticos necessários ao desenvolvimento do País e a melhoria da condição de vida do povo. A Geologia assume inquestionável relevância dentre as demais Ciências da Terra neste milênio, porque focaliza questões e recursos imprescindíveis para a sociedade e o desenvolvimento do País: a água de boa qualidade para abastecimento humano, as fontes de energia, o suprimento de matérias primas minerais, o planejamento urbano, a problemática complexa dos riscos ambientais e outros temas relacionados com a interação entre as sociedades e o meio ambiente. A própria pesquisa científica de Marte e outros corpos do Sistema Solar ou fora dele (Requeijo \& Carneiro 2010), encontra-se firmemente assentada sobre fundamentos de geologia planetária.

Roteiros de campo como o publicado por Uhlein e colaboradores constituem contribuição inestimável para atender à preocupação de docentes e coordenadores de curso com a quantidade e a qualidade das atividades de campo (Nummer et al. 2005, Fantinel et al. 2008). A comunidade é praticamente unânime em relação à ideia de que as grades curriculares de todos os cursos devam incluir expressiva carga horária de trabalhos de campo, ainda que isso implique custos consideráveis para que as universidades implantem e mantenham os cursos. Outra questão que tem ocupado as coordenações de curso refere-se à segurança das atividades de campo, que jamais podem ser negligenciadas (The Geological Society 2012a, 2012b, Teixeira \& Abreu 2017).

Desde a Comissão de Especialistas de Ensino de Geologia e Oceanografia, cujo relatório foi apresentado ao MEC em 1999 (Santos et al. 1999), existe certo consenso sobre a importância do contato com a natureza (Carneiro 2015) para ajudar os geólogos a formar competências e habilidades relacionadas à observação criteriosa e ao trabalho incessante de investigação que abre as portas para construções mentais cada vez mais sofisticadas. Com certo exagero, alguns colegas afirmam que "Geologia se aprende pelos pés".

Nos campos da Geologia e Engenharia Geológica, as Diretrizes Curriculares Nacionais para os Cursos de Geologia e Engenharia Geológica (DCNGEG) começaram a ser estabelecidas em fins de 2012 quando o Conselho Nacional de Educação aprovou relatório do Prof. Dr. Luiz Roberto Liza Curi (Brasil, Ministério da Educação 2012). No início de 2015 o MEC instituiu as Diretrizes Curriculares Nacionais (Brasil, Ministério da Educação 2015) para tais cursos de graduação, abrangendo o bacharelado em Geologia e em Engenharia Geológica. Os documentos estabelecem que a carga horária mínima de trabalhos de campo não pode ser inferior a $20 \%$ da carga horária mínima de 3.600 horas de curso. Talvez uma das principais contribuições dos dois artigos publicados em Terræ Didatica seja a recomendação implícita para fortalecimento e especialização das atividades de campo na formação de profissionais das áreas ligadas às Ciências da Terra. 


\section{Centro de Geologia Eschwege}

O Centro de Geologia Eschwege dispõe de instrutores de alto nível e bem montada infra-estrutura acadêmica. Oferece ensino-aprendizagem de técnicas de mapeamento e investigação geológica a estudantes de todo o país, explorando muito bem sua imersão em uma das principais unidades geológicas do Brasil, a Serra do Espinhaço. A trajetória do Centro de Geologia Eschwege é descrita pelo sitehtt:Hesrom da seguinte forma:

Instituto Eschwege foi o nome inicial do Centro de Geologia Eschwege, um espaço de ciência referência no ensino de mapeamento geológico. Mantido inicialmente com recursos financeiros do governo alemão o Instituto funcionou na casa da Rua Direita, 36 (ao lado do Museu de Diamante), em 1972, foi adquirida a casa da Rua Silvério Lessa. Em 1978, quando cessou a subvenção alemã, a UFMG, através do Ministério da Educação, MEC, adquiriu os dois casarões interligados pelo passadiço para ali instalar o Instituto Eschwege, que, a partir desse momento, 1979, passou a se chamar Centro de Geologia Eschwege, CGE. O "Eschwege" se fixou na rotina do ensino da geologia dos cursos de graduação no Brasil e desempenhou papel fundamental para a formação de centenas de geólogos brasileiros e para a realização de mais de sete dezenas de teses de doutoramento e dissertações de mestrado defendidas em universidades brasileiras e alemãs.

O desafio da descoberta científica, a beleza cênica da região e sobretudo o interesse pela capacitação de alto nível de geólogos alemães e brasileiros motivaram equipes de geólogos, pesquisadores, professores e estudantes brasileiros e alemães a implantar o CGE na cidade de Diamantina (MG). Seu criador foi o professor Reinhard Pflug, graduado em Geologia na Universidade de Bonn em 1955, com o título de Doutor em Ciências Naturais pela mesma universidade em 1958. Professor da Escola Nacional de Geologia do Rio de Janeiro (atual Universidade Federal do Rio de Janeiro, UFRJ), Pflug pesquisou regiões do sul do Brasil e oeste mineiro, mas concentrou sua atenção e interesse pela Serra do Espinhaço Meridional, onde desenvolvera trabalhos de mapeamento geológico e estudos sedimentológicos, estratigráficos e estruturais. No site http://csr.ufmg.br/geoespinhaco/pesquisa.htm observamos que:
A experiência de Pflug no território mineiro, o apoio e reconhecimento que sempre obteve do DNPM e de geólogos eminentes como Othon Leonardos e, por fim, o seu status acadêmico em Heidelberg contribuíram para convencer os governos alemão e brasileiro sobre a pertinência de um projeto de colaboração entre os dois países para dar continuidade às pesquisas geológicas na Serra do Espinhaço.

Tivemos a oportunidade de participar, juntamente com o Prof. Dr. Miguel Ângelo Stipp Basei, ambos vinculados à $\mathrm{USP}_{2}$ das primeiras reuniões de coordenadores de cursos de graduação em Geologia e Engenharia Geológica, nos anos 1976 e 1977, quando se discutiram formas de incorporação do Centro de Geologia Eschwege pela Universidade Federal de Minas Gerais, diante do iminente corte da subvenção alemã. Os entendimentos chegaram a bom termo, pois a UFMG soube reconhecer a relevância do centro para a preparação e treinamento profissional e, com o tempo, expandiu as atividades até formar o atual centro de referência denominado Casa da Glória, instalada à Rua da Glória, 298, no centro de Diamantina. Uma marcante característica da edificação é o passadiço que interliga prédios dos dois lados da rua (visite: https://www.ufmg.br/dlo/ ICG_Casa_Gloria.html).

\section{A visão sistêmica dos trabalhos de campo}

Trabalhos de campo nas duas regiões podem explorar as belas paisagens locais para que os participantes sejam capazes de decompor a estrutura física, química e biótica da superfície do planeta e construir um novo olhar sobre a Terra, sob a moderna perspectiva dos geossistemas terrestres. Sob este ponto de vista, parte da energia interna do planeta e da energia recebida do Sol promovem interação com as demais esferas (ou geossistemas). Assim, a leitura das paisagens, das rochas e seus arranjos espaciais privilegia a base geológica (Carneiro \& Gonçalves 2011) como sendo a parte fundamental sobre a qual se vida e as feições da superfície terrestre, sempre em permanente transformação.

Trabalhos de campo caminham de mãos dadas com a preparação e treinamento profissional de geólogos, engenheiros geólogos e outros profissionais de Ciências da Terra. A ampla variedade de ambientes geológicos brasileiros e a enorme extensão territorial do País estimulam a proposição de outros roteiros, para que se somem aos citados. Fazemos votos de que os cursos nacionais 
encontrem uma posição de equilíbrio entre o custo muitas vezes proibitivo de trabalhos de campo em regiões distantes e o benefício pedagógico resultante das autênticas "aventuras geológicas".

\section{Referências}

Assine M.L. 1994. Repensando os cursos de graduação em Geologia. Cadernos IG-Unicamp, 4(1):34-65.

Azevedo R.L.M.de, Terra G.J.S. 2008. A busca do petróleo, o papel da Petrobras e o ensino da Geologia no Brasil. B. Geoci. Petrobras, 16(2):373-420.

Barroso J.A. 1996. Os 40 anos da CAGE: Campanha Nacional de Formação de Geólogos, os cursos de geologia no Brasil e, em particular, no Rio de Janeiro. Rio de Janeiro: Anuário Inst. Geoc. UFRJ, 19:143156. URL: http://www.ppegeo.igc.usp.br/index. php/anigeo/article/view/1744. Acesso 14.03.2018.

Brasil. Ministério da Educação. Conselho Nacional de Educação. Câmara de Educação Superior. 2012. Resolução CNE/CES n. 387/2012, de 7 novembro de 2012. Institui as diretrizes curriculares nacionais para o curso de graduação em Geologia e em Engenharia Geológica, bacharelados. Brasília (DF), 7 nov. 2012. (Relator Luiz Roberto Liza Curi, Proc. No: 23001.000110/2007-41).

Brasil. Ministério da Educação. Conselho Nacional de Educação. Câmara de Educação Superior. 2015. Resolução 1, de 6 de janeiro de 2015. Institui as Diretrizes Curriculares Nacionais para os cursos de graduação na área da Geologia, abrangendo os cursos de bacharelado em Geologia e em Engenharia Geológica e dá outras providências. Brasília (DF), 6 jan. 2015.

Carneiro C.D.R. 2015. Cursos de graduação em Geologia sem aulas de campo são inviáveis. In: Simp. Nac. Ens. Hist. Ciênc. Terra, 6, Simp. Geol. Sudeste, 14, Campos do Jordão, 26-29.10.2015. Anais... Campos do Jordão, SBGeo. p. 654-658. URL: www.sp.sbgeo. org.br/SBG-SP/Eventos_files/ANAIS-GEOSUDESTE-2015-completo.pdf. Acesso 08.08.2017.

Carneiro C.D.R., Gonçalves P.W. 2011. Actividades de campo en la asignatura Ciencia del Sistema Tierra: la Geología como estructura básica. Rev. de la Enseñanza de las Ciencias de la Tierra, 19(1):48-56. URL: http://www.raco.cat/index.php/ECT/article/ view/244378/331350. Acesso 20.03.2018.

Fantinel L., Janasi V.A., Assis J.F.P., Alecrim J.R., Almeida H.L.de, Compiani M., Conceição R., Duarte B.P., Fauth G., Fonseca V.P.da, Fortes P., Leite Júnior W.B., Mancini F., Menezes M.G.de, Silva C.H.da, Silva Filho W., Velloso E., Carneiro C.D.R. 2008. Diretrizes Curriculares para os Cursos de Graduação em Geologia e Engenharia Geológica. Terra Didatica, 4(1):85-89. URL: http://www.ige.unicamp. br/terraedidatica/v4/TD_4.html. Acesso 20.03.2018.

Ministério da Educação, MĒC. 1999. Comissão de Especialistas de Ensino de Geologia e Oceanografia, Portaria n 146 SESu/MEC de 10 de março de 1998,
Sub-Comissão de Geologia: Newton Souza Gomes, Paulo Milton Barbosa Landim e Reinhardt Adolfo Fuck. 1999. Diretrizes Curriculares para os Cursos de Graduação em Geologia e Engenharia Geológica. Brasília: MEC. 9p.

Nummer A.R., Godoy A.M., Lazzarotto A., Carneiro C.D.R., Schultz C.L., Tubbs Filho D., Guimarães E.M., Althoff F. Assis J.F.P., Pinho F.E.C. Sobreira F., Carvalho I.S., Sabadia J.A.B.Fernandes Filho L.A., Toledo M.C.M.de, Fernandes M.L.S., Costa R.D.da, Machado R., Menegat R., Nadalin R.J., Santos R.A.A.dos, Vasconcelos S.M.S., Marques T.M. Souza Z.S.de. 2005. Diretrizes Curriculares para os Cursos de Graduação em Geologia e Engenharia Geológica. Terre Didatica, 1(1):64-69. http:// www.ige.unicamp.br/terraedidatica/v1/v1_a8.html. Acesso 20.03.2018.

Requeijo F., Carneiro C.D.R. 2010. Tem alguém aí? Zonas habitáveis na Via Láctea. In: A. Ivanissevich, C.A. Wuensche, J.F.V.da Rocha. 2010. Astronomia Hoje. Rio de Janeiro: Inst. Ciência Hoje. p. 82-93.

Santos L.C.M.L., Aquino I.B.M., Nunes B.A. 2017. A Mina-Escola de Santa Luzia-PB: um laboratório didático para estudantes de Mineração/Geologia. Terrce Didatica, 13(3):244-257. DOI: 10.20396/ td.v13i3.8650960. Acesso 20.03.2018.

Santos M.I.F.dos, Gomes N.S., Landim P.M.B., Vieira P.J.C., Fuck R.A. 1999. Diretrizes Curriculares para os Cursos de Graduação em Geologia e Engenharia Geológica. Brasília: Ministério da Educação, Secr. Educ. Superior., Coordenadoria das Comissões de Especialistas de Ensino.

Sociedade Brasileira de Geologia (SBG). 1983. Simpósio Nacional sobre o Ensino de Geologia no Brasil. Currículo Mínimo, 2. 1982, Salvador. Documento Final... São Paulo: SBG. 73p.

Sociedade Brasileira de Geologia (SBG). 1984. A formação do geólogo nas universidades brasileiras: um retrato de duas décadas. São Paulo: SBG.

Uhlein A., Ersinzon F., Uhlein G.J., Alcântara D.G. 2017. Estratigrafia e sistemas deposicionais do Supergrupo Espinhaço e Grupos Bambuí e Macaúbas: roteiro de campo na Serra do Espinhaço Meridional (MG). Terre Didatica, 13(3):244-257. DOI: 10.20396/ td.v13i3.8650963. Acesso 20.03.2018.

The Geological Society. 2012a. Code for Geological Fieldwork. London: The Geol. Soc. URL: https://www. geolsoc.org.uk/FieldResources. Acesso 20.03.2018.

The Geological Society. 2012b. Geological Fieldwork Code. London: The Geol. Soc. URL: https://www.geolsoc. org.uk/ /media/shared/documents/education $\% 20$ and $\% 20$ careers/GARockCoringGuide.pdf?la $=$ en. Acesso 20.03.2018.

Teira 2017. Conceitos básicos sobre segurança em trabalhos de campo para cursos de graduação em Geologia e Engenharia Geológica. Terree Didatica, 13(3):323-331. DOI: 10.20396/td.v13i3.8651227. Acesso 20.03.2018. 